\title{
Evaluation of next generation sequencing platforms for population targeted sequencing studies Olivier Harismendy ${ }^{\mathfrak{\alpha}^{*}}$, Pauline $\mathrm{C} \mathrm{Ng}^{\star \dagger}$, Robert L Strausberg ${ }^{\dagger}$, Xiaoyun Wang ${ }^{*}$, Timothy B Stockwell ${ }^{\dagger}$, Karen Y Beeson ${ }^{\dagger}$, Nicholas J Schork*, Sarah S Murray*, Eric J Topol*, Samuel Levy ${ }^{\dagger}$ and Kelly A Frazer*
}

Addresses: *Scripps Genomic Medicine - Scripps Translational Science Institute - The Scripps Research Institute, N. Torrey Pines Court, La Jolla, CA 92037, USA. 'The J Craig Venter Institute, Medical Center Drive, Rockville, MD 20850, USA.

$\ltimes$ These authors contributed equally to this work.

Correspondence: Samuel Levy. Email: slevy@jcvi.org. Kelly A Frazer. Email: kfrazer@scripps.edu

Published: 27 March 2009

Genome Biology 2009, 10:R32 (doi:10.1 I86/gb-2009-10-3-r32)

The electronic version of this article is the complete one and can be found online at http://genomebiology.com/2009// 0/3/R32
Received: 14 December 2008

Revised: 23 February 2009

Accepted: 27 March 2009

(C) 2009 Harismendy et al.; licensee BioMed Central Ltd.

This is an open access article distributed under the terms of the Creative Commons Attribution License (http://creativecommons.org/licenses/by/2.0), which permits unrestricted use, distribution, and reproduction in any medium, provided the original work is properly cited.

\begin{abstract}
Background: Next generation sequencing (NGS) platforms are currently being utilized for targeted sequencing of candidate genes or genomic intervals to perform sequence-based association studies. To evaluate these platforms for this application, we analyzed human sequence generated by the Roche 454, Illumina GA, and the ABI SOLiD technologies for the same $260 \mathrm{~kb}$ in four individuals.
\end{abstract}

Results: Local sequence characteristics contribute to systematic variability in sequence coverage (>100-fold difference in per-base coverage), resulting in patterns for each NGS technology that are highly correlated between samples. A comparison of the base calls to $88 \mathrm{~kb}$ of overlapping $A B I$ $3730 x \mathrm{~L}$ Sanger sequence generated for the same samples showed that the NGS platforms all have high sensitivity, identifying $>95 \%$ of variant sites. At high coverage, depth base calling errors are systematic, resulting from local sequence contexts; as the coverage is lowered additional 'random sampling' errors in base calling occur.

Conclusions: Our study provides important insights into systematic biases and data variability that need to be considered when utilizing NGS platforms for population targeted sequencing studies.

\section{Background}

The Sanger method [1] of sequencing by capillary electrophoresis using the ABI 3730xL platform has been employed in many historically significant large-scale sequencing projects and is considered the 'gold standard' in terms of both read length and sequencing accuracy [2]. Several next generation sequencing (NGS) technologies have recently emerged, including Roche 454, Illumina GA, and ABI SOLiD, which are able to generate three to four orders of magnitude more sequence and are considerably less expensive than the Sanger 
method on the ABI 3730xL platform (hereafter referred to as ABI Sanger) [2-4]. To date these new technologies have been successfully applied toward ChIP-sequencing to identify binding sites of DNA-associated proteins [5,6], RNAsequencing to profile the mammalian transcriptome $[7,8]$, as well as whole human genome sequencing [9-11]. Currently there is much interest in applying NGS platforms for targeted sequencing of specific candidate genes, intervals identified through single nucleotide polymorphism (SNP)-based association studies, or the entire human exome [12-15] in large numbers of individuals.
As population targeted sequencing studies are initiated, it is important to determine the issues that will be encountered in generating and analyzing data produced by NGS platforms for this application. Here, we generate $260 \mathrm{~kb}$ of targeted sequence in four samples using the manufacturer recommended and/or supplied sample library preparation methods, sequence generation, alignment tools, and base calling algorithms for the Roche 454, Illumina GA, and ABI SOLiD platforms (Figure 1). For each NGS technology we generated a saturating level of redundant sequence coverage, meaning that increased coverage is likely to have minimal, if any, effect on data quality and variant calling accuracies. We analyzed the sequences produced by each platform for per-base



\section{Figure I}

Overview of experimental design. Six genomic intervals, each encoding genes for $\mathrm{K}^{+} / \mathrm{Na}^{+}$voltage-gated channel proteins, were amplified using DNA from four individuals and LR-PCR reactions to generate $260 \mathrm{~kb}$ of target sequence per sample. Amplicons from each individual were pooled in equimolar amounts and then sequenced using the three NGS platforms. The $260 \mathrm{~kb}$ examined in this study is representative of human sequences containing $38 \%$ repeats and $4 \%$ coding sequence compared with $47 \%$ and I\%, respectively, genome-wide. For each sample $88 \mathrm{~kb}$ was amplified using short range PCR (SRPCR) reactions targeting the exons and evolutionarily conserved intronic regions. Each SR-PCR amplicon was individually sequenced in the forward and reverse directions using the ABI-3730xL platform (Additional data file 2). Data generated from the NGS platforms were analyzed to identify bases variants from the reference sequence (build 36) and the quality of the variant calls was assessed using platform specific methodologies. A comparative analysis of the sequence data from the NGS platforms and ABI Sanger was then performed to determine accuracy, and false positive and false negative rates. 
sequence coverage and for systematic biases giving rise to low coverage. We show that each NGS platform generates its own unique pattern of biased sequence coverage that is consistent between samples. For the short-read platforms, low coverage intervals tend to be in AT-rich repetitive sequences. We also performed a comparative analysis with sequence generated by the well-established ABI Sanger platform (Figure 1) to determine base calling accuracies and how average fold sequence coverage impacts base calling errors. Although the three NGS technologies correctly identify $>95 \%$ of variant alleles, the average sequence coverage required to achieve this performance is greater than the targeted levels of most current studies.

\section{Results \\ Generation and alignment of sequence reads to targeted intervals}

The targeted sequence was amplified in the four DNA samples using long-range PCR (LR-PCR) reactions that were combined in equimolar amounts and sequenced using the three NGS technologies (Figure 1). For the Roche 454 platform we obtained an average of 49,00o reads per sample with an average length of 245 bp (Supplemental Table 1 in Additional data file 1), using Illumina GA we generated an average of 5.9 million reads each 36 bases in length per sample, and using ABI SOLiD we obtained an average of 19.7 million reads each 35 bases in length per sample. Thus, the amount of sequence data generated and analyzed was dependent on the NGS platform and the fraction of the run that was utilized.

The NGS technologies generate a large amount of sequence but, for the platforms that produce short-sequence reads, greater than half of this sequence is not usable. On average, $55 \%$ of the Illumina GA reads pass quality filters, of which approximately $77 \%$ align to the reference sequence (Supplemental Table 1 in Additional data file 1; Additional data file 2). For ABI SOLiD, approximately $35 \%$ of the reads pass quality filters, and subsequently $96 \%$ of the filtered reads align to the reference sequence. Thus, only $43 \%$ and $34 \%$ of the Illumina GA and ABI SOLiD raw reads, respectively, are usable. In contrast to the platforms generating short-read lengths, approximately $95 \%$ of the Roche 454 reads uniquely align to the target sequence. When designing experiments and calculating the target coverage for a region, one must consider the fraction of alignable sequence.

\section{Overrepresentation of amplicon end sequences}

In examining the distribution of mapped reads, we observed that the sequences corresponding to the $50 \mathrm{bp}$ at the ends and the overlapping intervals of the amplicons have extremely high coverage (Figure 2; Additional data file 2). These regions, representing about 2.3\% (approximately $6 \mathrm{~kb}$ ) of the targeted intervals, account for up to $56 \%$ of the sequenced base pairs for Illumina GA technology. This extreme sequence coverage bias results from overrepresentation of the ampli- con ends in the DNA samples after fragmentation prior to library generation. For the ABI SOLiD platform an amplicon end depletion protocol was employed to remove the overrepresented amplicon ends; this was partially successful and resulted in the ends accounting for up to $11 \%$ of the sequenced base pairs. For the Roche 454 technology, overrepresentation of amplicon ends versus internal bases is substantially less, with the ends composing only $5 \%$ of the total sequenced bases; this is likely due to library preparation process differences between Roche 454 and the short-read length platforms. The overrepresentation of amplicon end sequences is not only wasteful for the sequencing yield but also decreases the expected average coverage depth across the targeted intervals. Therefore, to accurately assess the consequences of sequence coverage on data quality, we removed the $50 \mathrm{bp}$ at the ends of the amplicons from subsequent analyses.

\section{Sequence coverage of targeted intervals}

For each platform we generated a saturating level of redundant sequence coverage, meaning that increased coverage is likely to have minimal, if any, effect on data quality. For the four samples the average sequence coverage depth across the analyzed base pairs is $43 \times, 188 \times$, and $841 \times$ for Roche 454 , Illumina GA, and ABI SOLiD, respectively (Supplemental Table 2 in Additional data file 1). For all three NGS technologies there is greater than a hundred-fold variation in the perbase sequence coverage depth (Figure 2). We performed several analyses to determine if the sample preparation method and/or a specific class of sequence elements were responsible for the observed variability (Additional data file 2). We first tested whether the large variability resulted from pooling of the amplicons. For $90 \%$ of the amplicons the fold difference in average coverage of unique sequences is less than 2.46, 2.72, and 2.99 on the Roche 454, Illumina GA and ABI SOLiD platforms, respectively (Supplemental Table 3 in Additional data file 1), showing that the error in equimolar pooling or amplicon specific bias (sequence, length) explains only a small fraction of the observed coverage variability. Next we examined how the sequence coverage differs within the individual amplicons. For Roche 454, Illumina GA, and ABI SOLiD the average coefficient of variance was $0.33,0.9$, and 0.73 , respectively, for all base pairs, and $0.35,0.84$ and 0.76 , respectively, when restricted to unique non-repetitive sequence, defined here as not present in the RepBase database [16]. These results indicate that unique sequences present at equimolar amounts in the library generation step end up being covered at vastly different read depths.

It is important to consider how well the NGS technologies are able to generate sequence reads containing repetitive elements as these sequences comprise approximately $45 \%$ of the human genome and may potentially impact genome function. Compared to unique sequences, the Roche 454 technology has a 1.25 -fold overrepresentation of LINE elements, Illumina GA has greater than 2-fold higher coverage of SINEs, Alus and simple repeats, while for ABI SOLiD all repetitive 


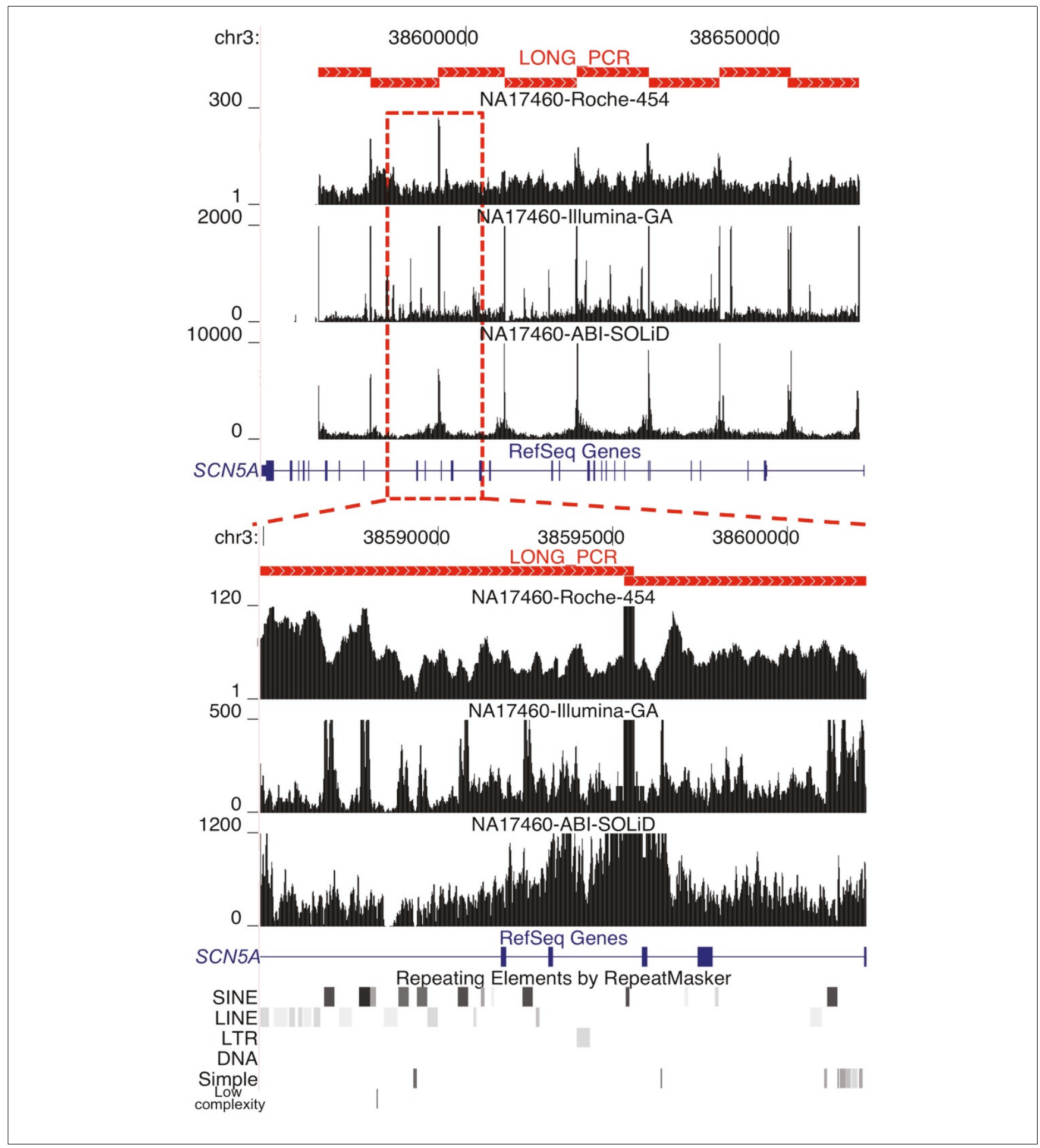

Figure 2

Non-uniform per-base sequence coverage. The 100-kb interval on chromosome 3 encoding the SCN5A gene (blue rectangles and joining lines) was amplified using eight LR-PCR amplicons (red filled rectangles in upper panel). On the $y$-axis, the fold sequence coverage scale is shown for each platform. The upper panel shows that amplicon end sequences are highly overrepresented. The $y$-axis was set to show the relative fold coverage of the sequences in the interval and therefore does not accurately represent the maximum fold coverage of the amplicon ends, which was $31 \mathrm{I}$, 195,473, and I5,04I for Roche 454, Illumina GA, and ABI SOLiD, respectively, in the sample shown. The lower panel shows the non-uniformity of sequence coverage across an approximately $17-\mathrm{kb}$ region encompassing four exons of SCN5A. The locations of the repetitive elements (lower black/gray rectangles) in the interval are shown. 
elements are covered at approximately half the fold coverage of unique sequences (Supplemental Table 4 in Additional data file 1). Thus, considering all three NGS platforms, Roche 454 generates the most even coverage across both unique and repetitive sequences, Illumina GA shows the most variability in coverage, and ABI SOLiD demonstrates a strong bias against coverage of repetitive elements.

Interestingly, each NGS technology has a unique reproducible pattern of non-uniform sequence coverage: sequences with high or low coverage in one sample typically had high or low coverage in the other three samples (Figure 3). The coefficient of correlation $(r)$ of per-base sequence coverage depth was 0.62 , 0.90, and 0.88 between samples on Roche 454, Illumina GA, and ABI SOLiD, respectively. On the other hand, per-base sequence coverage depth for the same sample on different platforms was not well correlated $(r<0.19)$. These data indicate that for all three NGS technologies local sequence characteristics substantially contribute to the observed variability in coverage unique to each technology.

To gain insight into systematic biases of each NGS technology, we examined the sequence composition of intervals with no or low coverage (defined as less than $5 \%$ of the average coverage depth; Additional data file 2). Despite having considerably higher average sequence coverage, the ABI SOLiD data have the largest number of no and low coverage intervals (spanning $464 \mathrm{bp}$ and 3,415 bp respectively), the majority of which are AT-rich repetitive sequences (Supplemental Tables 5 and 6 in Additional data file 1). The Illumina GA low coverage regions (spanning $272 \mathrm{bp}$ ) also tend to be AT-rich repetitive sequences. Overall, for the short read platforms read depth coverage decreases with increasing AT content, which is consistent with previous studies [17,18] (Supplemental Figure 1 in Additional data file 3). Roche 454 had one no and one low coverage interval (spanning $4 \mathrm{bp}$ and $59 \mathrm{bp}$, respectively).

\section{Detection of single nucleotide base variants}

We established parameters for calling variant bases in the sequence generated by the NGS technologies based on optimized concordance with the variant calls in the ABI Sanger data. As previously observed, PCR sample preparation can produce imbalanced amplification of the two alleles for some amplicons, resulting in incorrect genotype calls at variant bases by specifically calling heterozygous sites as homozygous sites [19]. Imbalanced amplification is usually suspected to result from polymorphisms in or near the oligonucleotide priming sites that result in greater efficiency of amplification for one of the alleles. To measure this phenomenon in our sample preparation method, we looked at the alternate allele read frequency (AARF; Additional data file 2) at ABI Sanger identified heterozygous positions in the sequence data for the three NGS platforms. Out of the 28 amplicons in this study, four demonstrated allelic imbalances in amplification for one or more samples (Supplemental Table 7 in Additional data file 1). We removed the sequence

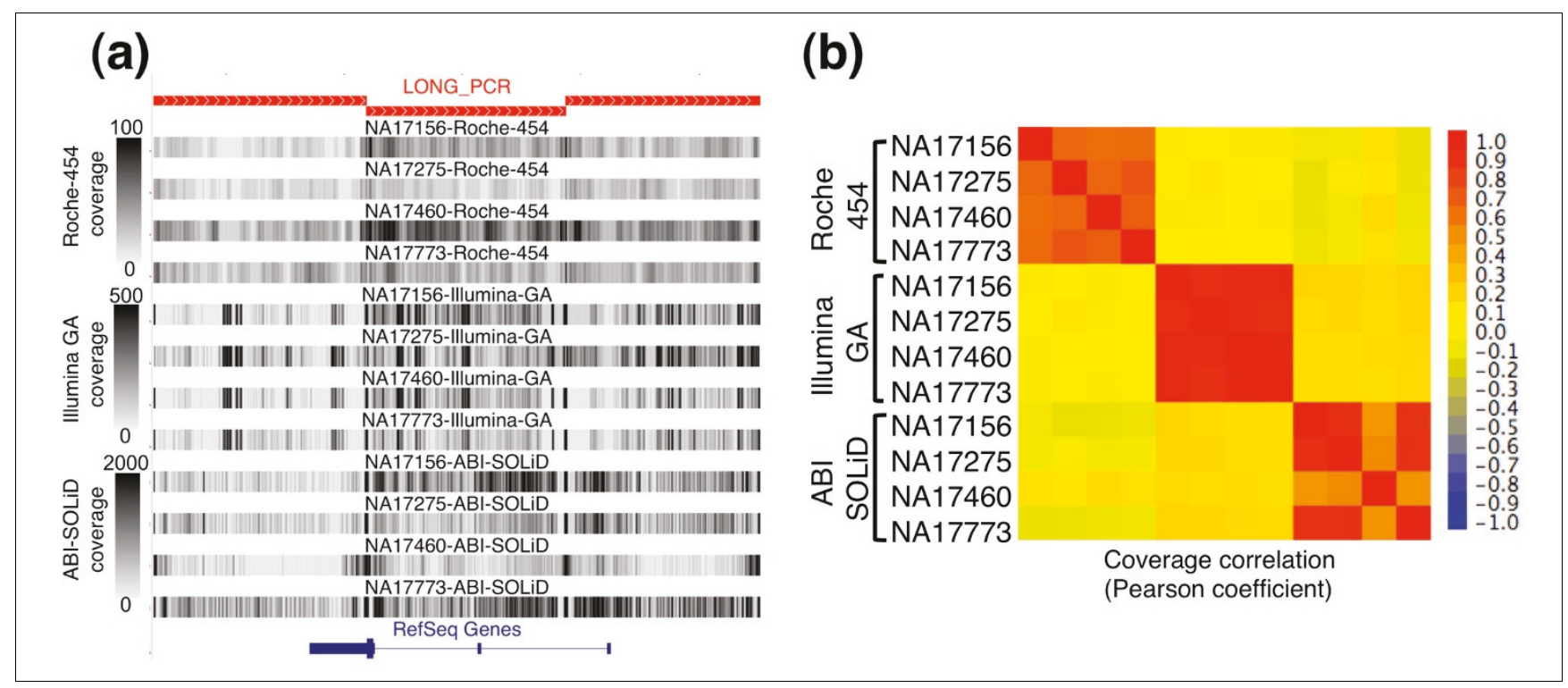

Figure 3

Each NGS technology generates a consistent pattern of non-uniform sequence coverage. (a) Sequence coverage depth is displayed as a gray-scale (0-100x for Roche 454; 0-500× for Illumina GA and ABI SOLiD) along an approximately 25-kb region of chromosome II amplified by three long-range PCR products (red rectangles). (b) A heat-map colored matrix displays the coefficient of correlation of coverage across the entire $260 \mathrm{~kb}$ of analyzed sequence between each of the 72 possible pair-wise comparisons (four samples by three technologies). The apparent lower correlation of the Roche-454 sequence coverage is more reflective of the smaller amplitude in the coverage variability (lower average coefficient of variance) than a lack of coverage correlation from sample to sample. The correlation of NAI7460 with the other three samples on the ABI SOLiD platform is slightly lower due to technological issues (Additional data file 2) and was therefore excluded from the coefficient of correlation calculation reported in the text. 
data for these four amplicons from the variant quality analysis so as to focus on errors caused by the NGS platforms and thereby not have the analysis confounded by sample preparation issues.

\section{Accuracy of sequence variant calls compared to microarray genotype calls}

Accuracy of the variant calls in the NGS and ABI Sanger data for the four samples was initially assessed by comparison to genotype calls for approximately 80 SNPs located in the sequenced intervals and assayed by the Illumina Hap550 BeadChip. The genotype accuracy of the four platforms is 97.4\%, 100\%, 99.7\%, and 98\% for Roche 454, Illumina GA, ABI SOLiD and ABI Sanger, respectively (Supplemental Tables 8 and 9 in Additional data file 1). These data show a greater number of discordant genotypes for Roche 454. It is important to note that comparison between sequence and SNPs genotyped on commercial arrays is not expected to be fully indicative of NGS platform variant base calling accuracy in genomic sequences at large. First, false positive rates cannot be considered by SNP microarray technologies because novel variants are not detected. Second, SNP microarrays typically query a subset of 'well behaved' bases; hence, false negative rates based on microarray technology can be underestimated.

\section{Variant detection comparing NGS to ABI Sanger}

To further assess sequence quality, we next performed a fourway comparison of the base calls generated from the three NGS technologies and ABI Sanger. The identification of heterozygous and homozygous alternate loci was performed in 258,879 base pairs analyzed from all four samples (Supplemental Table 10 in Additional data file 1). There were twenty loci for which the three NGS technologies were concordant in their base calls but discordant with the ABI Sanger calls. Visual inspection of the ABI Sanger traces revealed that eight of these loci represented base calling errors in the original data, thereby resolving the discrepancy. However, for 12 loci (9 false positive and 3 false negative calls) the discrepancies were not resolved (Figure 4g,h). Two of the discrepant calls were assayed by the Illumina Hap550 array (Supplemental Table 9 in Additional data file 1) and their calls were concordant with the NGS platforms. We examined the genotypes of the remaining discrepant calls by independent Sanger sequencing. As previously established [19,20], errors in Sanger sequencing of human diploid DNA are approximately $7 \%$ and result from: PCR primers sometimes overlapping unknown DNA variants leading to imbalanced amplification of the two alleles; and difficulty of automated software to correctly call heterozygous sites. Thus, replicating the Sanger sequencing with different PCR and sequencing primers and manual inspection of the traces can be considered an independent measurement. We successfully examined eight of the discrepant calls using this approach, of which seven agreed with the calls made by the NGS platforms (Supplemental Figure 3 in Additional data file 3). In total, nine of the ten dis- crepant calls investigated (two by genotyping and seven by Sanger sequencing) were confirmed as being incorrect in the original ABI-Sanger sequencing. As a result of this analysis for the first time by comparison with NGS technologies, the ABI Sanger false positive and false negative rates for human diploid DNA are estimated to be approximately $0.9 \%$ and approximately 3.1\%, respectively. These 12 loci identified as ABI Sanger errors were removed from consideration when assessing the NGS technologies' performance.

We next calculated five different performance metrics (sequencing accuracy, variant accuracy, false positive rate, false negative rate, and variant discrepancy rate) for the NGS platforms (Supplemental Table 11 in Additional data file 1). Sequencing accuracy, which measures the concordance of all calls including homozygous reference, was greater than 99.99\% for all NGS technologies (Figure 4a). On the other hand, variant accuracy, which measures the ability of NGS technologies to make a correct call at known variant positions identified by ABI Sanger, was lower, averaging over the four individuals for each technology at $95 \%, 100 \%$, and $96 \%$ for Roche 454, Illumina GA, ABI SOLiD, respectively (Figure 4b). The false positive rate of Roche 454, Illumina GA and ABI SOLiD is approximately $2.5 \%$, approximately $6.3 \%$, and approximately $7.8 \%$, respectively; the false negative rates are approximately $3.1 \%$, approximately $0 \%$, and $0.9 \%$ (Figure 4d,e). We also examined the variant discrepancy rates, which reflect the number of positions that have been correctly identified as variant, but assigned incorrect zygosity. For Roche 454, Illumina GA, and ABI SOLiD the variant discrepancy rates were $2 \%, 0 \%$, and $3 \%$, respectively. These five performance metrics indicate that at saturating sequence coverage and the methodologies employed to call variants, the shortread platforms have greater sensitivity but lower specificity than Roche 454 .

In examining the sequences underlying false positive and false negative calls in the NGS technologies, we determined that these errors were unexpectedly not associated with low sequence coverage but rather are the result of systematic biases (Figure 4g,h,i). For each NGS platform, 47\% of the bases with an error in one sample had an error in at least one other sample (Supplemental Table 12 in Additional data file 1). Greater than $72 \%$ of these false positive and negative calls are associated with at least one and $>33 \%$ with two of the following sequence contexts: repetitive elements; a homopolymer stretch $\geq 6$ bases; simple repeats; the presence of an indel within $30 \mathrm{bp}$. These sequence contexts likely present significant challenges during read alignment, especially for the short-read technologies, resulting in variant detection errors. Two out of the three false negatives specific for the ABI SOLiD platform were due to the inability to detect adjacent SNPs with existing variant calling software applied to color-space sequencing technology (Additional data file 2). 
(a) Sequencing accuracy

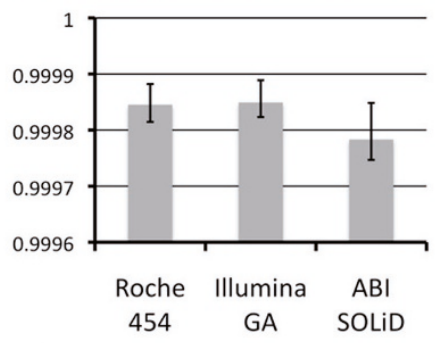

(d)

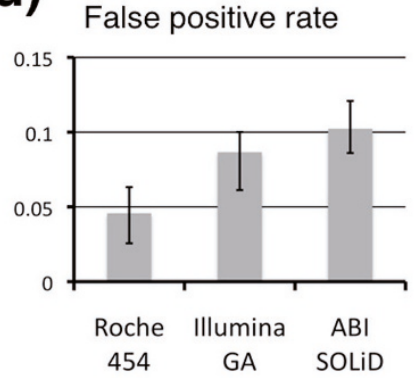

(g)



$\overrightarrow{1}+$

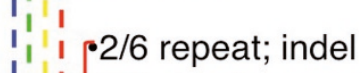

: $: 3 / 6$ simple repeat; indel

: $1: 1 / 11$ repeat

, $1 / 11$ simple repeat

I - 2/11 homopolymer

-4/11 homopolymer; indel

, $2 / 11$ indel

$\cdot 2 / 9$ repeat; indel

I'- - -1/9 homopolymer; indel

-6/9 simple repeat; indel

$[\cdot 1 / 18$ repeat

-2/18 homopolymer

ᄂ - $-\cdot 2 / 18$ indel

-5/18 simple repeat; indel

-2/18 homopolymer; indel (b) Variant accuracy

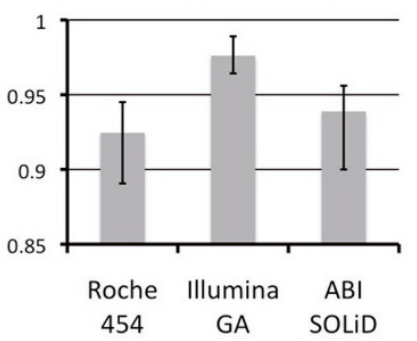

(e)

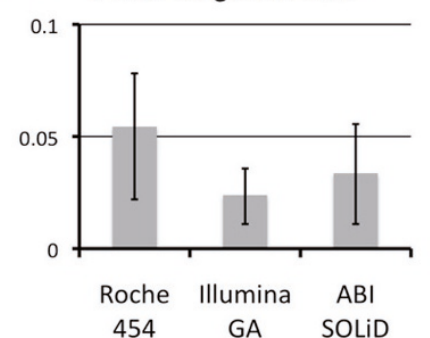

(h)

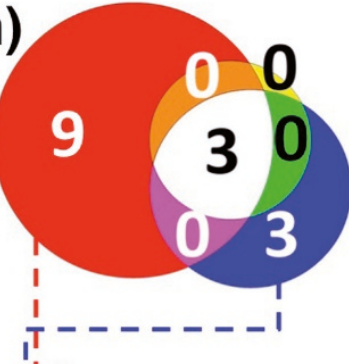

$1 / 9$ simple repeat -2/9 homopolymer e4/9 inde

I. $[-1 / 3$ repeat

$-2 / 3$ adjacent variants (c) Coverage rate

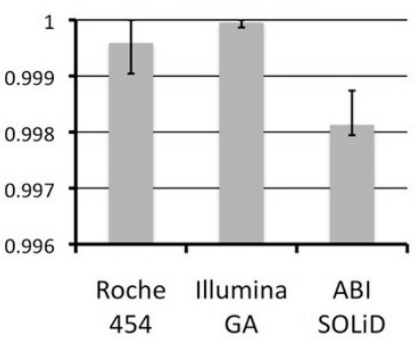

(f)

Variant discrepancy rate

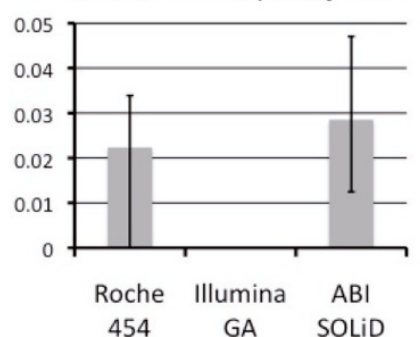

(i)

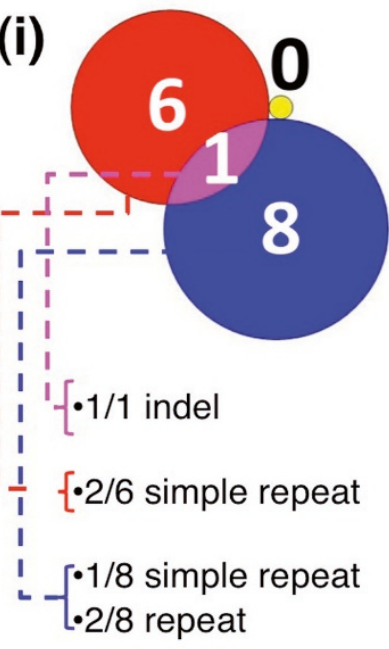



\section{Figure 4}

Performance metrics of NGS technologies. (a-f) Error bars represent minimum and maximum values obtained from the four samples. (g-i) Venn diagram representation of false positive calls (g), false negative calls $(\mathrm{h})$ and discrepant variants calls (i). The inset caption displays the color-coding of each NGS technology and overlaps: for Roche 454 (red), Illumina GA (yellow) and ABI SOLiD (blue). For each NGS platform the number of base calls with errors associated with specific sequence contexts is given (repeat = repetitive element). When two sequence contexts are present they are both listed. 


\section{Detection of indels}

Detection of heterozygous indels remains a technological challenge using the ABI Sanger platform [21]. Here the ABI Sanger sequencing detected 11 heterozygous indels in the 88 $\mathrm{kb}$ of sequence analyzed. The Roche 454 technology successfully identified five of these indels, all of which ranged from 3$16 \mathrm{bp}$ in length (Supplemental Table 13 in Additional data file 1). Of the six indels missed by Roche 454 , five were single base in length in homopolymer sequences, and one was a $15 \mathrm{bp}$ insertion that was not completely resolved due to low coverage. Interestingly, Roche 454 identified 43 additional indels in the $88 \mathrm{~kb}$ of overlapping ABI Sanger sequences (Supplemental Table 14 in Additional data file 1). Bearing in mind that the false positive rate for these data cannot be estimated, this suggests that the Roche 454 platform may be more useful for identifying indels than the ABI Sanger technology. The Illumina GA and ABI SOLiD platforms at the time of this analysis were unable to identify indels automatically.

\section{Assessing performance metrics at lower coverage}

To efficiently perform population-based targeted sequencing studies using NGS technologies, it is important to determine the lowest average sequence coverage required to achieve a specified sensitivity and specificity. To estimate this coverage requirement, we simulated varying coverage depths for all three technologies, recalled genotypes, and calculated false positive and false negative rates for each coverage depth (Additional data file 2). The maximum simulated average coverage was 40-fold for Roche 454 and 140-fold for both Illumina GA and ABI SOLiD. The false positive error rates are more impacted by low coverage compared with false negative rates; thus, we focused our analysis on the former. The average coverage depth for $50 \%$ false positive error rate degradation (percentage of the minimum simulated error rate; see
Materials and methods) is achieved at 25-fold, 68-fold, and 39 -fold and for $10 \%$ degradation at 34-fold, 110-fold and 101fold for Roche 454, Illumina GA, and ABI SOLiD, respectively (Figure 5). These results indicate that the short-read technologies have a two- to three-fold greater sequence coverage depth requirement relative to Roche 454 . Thus, errors at high coverage are systematic and typically associated with specific sequence contexts; at lower coverage errors result from random sampling in base calling. Consistent with this observation, the performance of the NGS technologies at low sequence coverage is correlated with per-base sequence coverage uniformity; the Illumina GA, which has the highest coverage variability, performs the worst at lower coverage, whereas Roche 454, with the most uniform coverage, performs the best. This observation suggests that for all the NGS technologies, achieving more uniform sequence coverage would result in considerably higher performance at lower coverage.

\section{Discussion}

Our study highlights many issues encountered as NGS platforms are utilized for population-based targeted sequencing studies, including biases in sample library generation, difficulties mapping short reads, variation in sequence coverage depth of unique and repetitive elements, difficulties detecting indels with short reads, the systematic errors of the NGS technologies and the impact of all these features on variant calling accuracy. We note that the results of our analyses reported for each NGS platform are the combined effects of the manufacturer recommended laboratory methods, sequence read alignment tools, and base calling algorithms utilized.

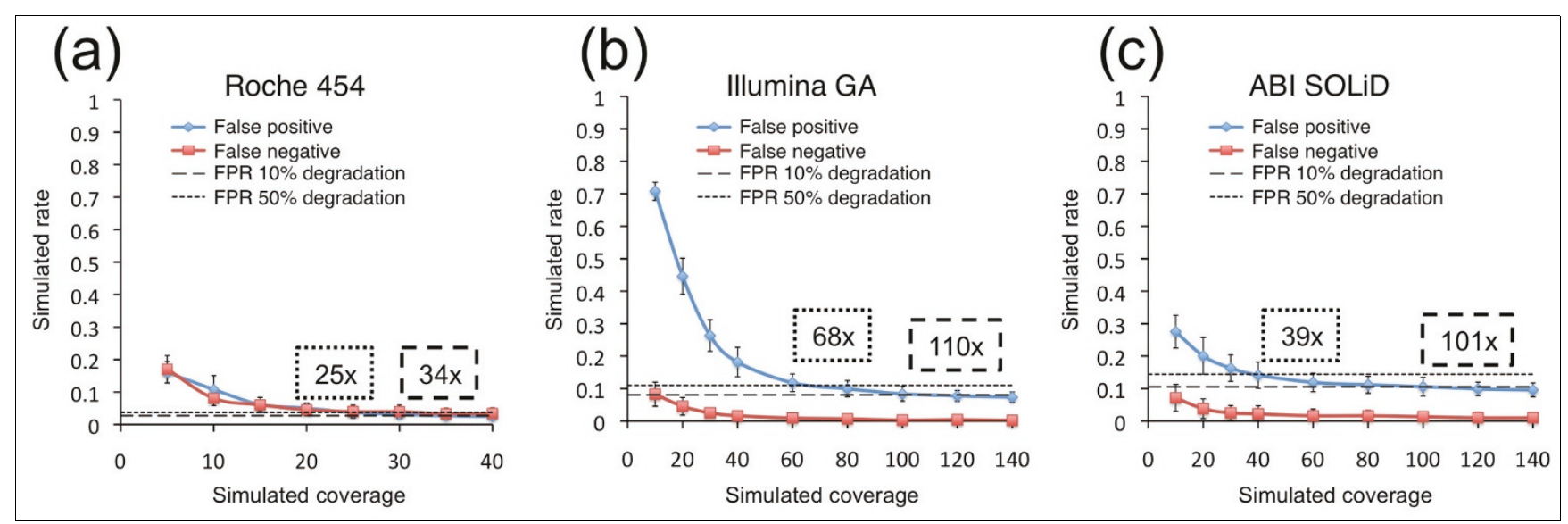

Figure 5

False positive rates (FPRs) and false negative rates for the three NGS technologies at simulated varying coverage depths. Performances of (a) Roche 454, (b) Illumina GA, and (c) ABI SOLiD at lower coverage depths were simulated by random subsampling of the reads. Error bars represent the standard deviation over the four samples for ten iterations. The thresholds for a $10 \%$ and $50 \%$ error rate degradation of the minimum false positive rate are indicated by dashed and dotted lines, respectively, and the corresponding coverage depth reported in dashed and dotted boxes, respectively. 
At high sequence coverage all NGS platforms have excellent variant calling accuracy ( $>95 \%$ ) as assessed by the detection of known SNP variants. However, this accuracy is lower than the values typically stated for the NGS platforms [22-25]. NGS-reported accuracies are typically being measured, in human sequences, by comparison to commercial SNP genotyping arrays, which we demonstrate are inadequate for ascertaining false positive and false negative rates. Therefore, the sequence-based accuracies reported here are likely to be more indicative of the real performance of NGS platforms for de novo detection of variants in human sequences.

Interestingly, our analysis indicates that ABI Sanger has a false negative rate of approximately $3 \%$, which is comparable to the three NGS technologies at saturating coverage. Thus, there are likely many more DNA polymorphisms yet to be detected in human samples [26]. Indeed, heterozygous indel detection, which is difficult using PCR-based sample preparation methods and ABI Sanger sequencing [27], may be easier to achieve using NGS platforms because each allele is sequenced and detected independently. This is especially important since indel variants constitute approximately $25 \%$ of the reported mutations implicated in human disease [28] and their identification would precede a more complete understanding of how they determine human phenotypes.

The saturating sequencing coverage we exploited enabled the determination of the sequence coverage threshold below which false discovery rates of variants were unacceptably high. This revealed that for accurate detection of biallelic sites, the average depth of sequence coverage required for all three NGS platforms but especially for the short-read technologies is considerably higher than the empirically determined coverage of 20-fold utilizing random Sanger sequencing [29]. This coverage requirement for NGS technologies is further supported by a recent multiplexed targeted resequencing study that showed that accurate detection of variant loci necessitates a 20-fold read depth per base, and a higher average depth due to coverage variability [30], and a recent yeast mutational profiling study that showed 10-15fold coverage is required to detect variants in haploid organisms [31]. Importantly, these required average sequence coverages are much higher than what is typically employed in targeted sequencing studies utilizing NGS technologies.

\section{Conclusions}

Our results suggest that to effectively balance cost and data quality for population targeted sequencing studies, there are two key aspects of NGS technologies that need optimization: the uniformity of per-base sequence coverage must be improved to reduce the total amount of sequence generation required; and the systematic errors that impact variant calling accuracy need to be reduced so that the false positive and false negative rates are acceptable for sequence-based association studies. Although recent improvements in the NGS platforms, such as paired end and longer reads, will mitigate these issues, all aspects of the NGS platforms, laboratory methods, sequence alignment tools, and base calling algorithms partially contribute to the problems and, therefore, need to be simultaneously optimized.

\section{Materials and methods Sample preparation}

Twenty-eight LR-PCR reactions were performed to amplify six genomic intervals spanning a total of $266 \mathrm{~kb}$ in each of four DNA samples (NA17275, NA1746o, NA17156, and NA17773) obtained from the Coriell Institute [32] (Additional data file 2). Following LR-PCR, the 28 amplicons generated using a single DNA sample template, ranging in size from $3,088 \mathrm{bp}$ to $14,477 \mathrm{bp}$, were quantified, combined in equimolar amounts, and used to create libraries for Roche 454, Illumina GA and ABI SOLiD sequencing.

\section{Roche 454}

The Roche 454 laboratory methods and protocols used were as described by Rothberg and coworkers [23]. The reads produced by the Roche 454 FLX platform were mapped to the reference sequence using the algorithm Newbler version 1.1.03.19 (provided by Roche), unless stated otherwise.

\section{Illumina GA}

The Illumina GA libraries were prepared according to the manufacturer's instructions from the 28 equimolar pooled PCR products except for the fragmentation step (Additional data file 2). The Illumina GA reads were aligned with MAQ o.6.2 [33], unless stated otherwise.

\section{ABI SOLiD}

Long mate pair (LMP) libraries DNA libraries were generated from the four 28 equimolar pooled amplicon samples and end sequenced using standard ABI SOLiD protocols at Applied Biosystems in Beverly, MA. For each sample, ABI aligned the sequence reads to the reference sequence and mate-pairing information was not employed in this project. The aligned reads and the number of calls per base for each position were used for data analysis (Additional data file 2).

The LMP library construction process requires more DNA amplification and manipulation and is useful for the detection of indels and structural variants. Therefore, as opposed to the library construction processes for Roche-454 and Illumina GA, which were focused on read fragment preparation alone, discarding mate-pair information from the LMP protocol reads and using them as unpaired reads may have introduced mapping biases when used to detect SNPs. Indeed, the generation of these libraries creates variable tag lengths that require different mapping techniques to ensure proper representation of the genome. Shorter tags will not map with a 35 bp and 3 mismatches schema and as a result substantial por- 
tions of the genome can be differentially sampled due to fixed mapping criteria.

These differences in the library techniques emphasize the need for the use of quality score information in the ABI SOLiD reads to properly trim the data before mapping and allow for proper comparison to a Roche 454 and Illumina GA data that currently perform Keypass, Chastity and Purity filtering of the data before SNP calling.

\section{Calling genotypes in the NGS sequence data}

We define the alternate allele as the most commonly called base (which is not the reference base) for a given position in the reference sequence. Then, the AARF is the fraction of reads corresponding to the alternate allele.

Positions called as reference homozygote by ABI Sanger have AARFs close to o\% by the NGS technologies (Supplemental Figure 2 in Additional data file 3). Also, positions called as alternate homozygous by ABI Sanger have AARFs near or at $100 \%$ by the NGS technologies. The AARFs for heterozygous calls by ABI Sanger is centered at 50\% for Roche 454 and Illumina GA; for ABI SOLiD it is centered at $42 \%$ (Additional data file 2). Upon independent inspection of the three technologies, most ABI Sanger-called heterozygotes fell in the range $20-80 \%$. Thus, for the NGS technologies, utilizing only high quality bases we call positions with AARFs between 20\% and $80 \%$ as heterozygous, positions with AARFs $>80 \%$ as homozygous alternate, and positions with AARFs <20\% as homozygous reference (Additional data file 2).

\section{Short-range PCR and Sanger sequencing}

We used an existing data set deposited by JCVI and performed under the auspices of the National Heart, Lung and Blood Re-sequencing and Genotyping program [34]. The data set included $88 \mathrm{~kb}$ of non-contiguous sequence encompassing the exons and the intronic sequence conserved with mouse and rat in the $\mathrm{K}^{+} / \mathrm{Na}^{+}$channel proteins produced by employing 273 short-range PCR reactions generating amplicons averaging $418 \mathrm{bp}$ in length.

\section{Definitions of performance metrics}

In order to assess the performance of the sequencing technologies, we define several metrics.

\section{Comparing a genotyping microarray to a sequencing technology}

Genotype accuracy

We genotyped the four samples on the Illumina Hap550 microarray according to specifications of the manufacturer. We compared the genotype calls of the SNPs on the Hap550 microarray with the genotypes observed from sequencing (Supplemental Table 8 in Additional data file 1). Genotype accuracy is defined as: (Number of genotypes matching exactly between Illumina Hap550 and a sequencing technology)/(Number of compared positions).

Metrics for comparing a NGS sequencing technology with ABI Sanger We initially assumed the ABI Sanger sequence data are correct because it is an established method with the longest history [2]. Upon further analysis, we found that this assumption was not always true; there were some positions incorrectly called by ABI Sanger, but correctly called by the NGS technologies (see Results). We refer to Table 1 annotations to clarify these definitions.

\section{Sequencing accuracy}

This is defined as the number of concordant calls between ABI Sanger and a NGS technology. Following the diagram above, this is calculated as $\left(\mathrm{A} 1+\mathrm{B}_{2}+\mathrm{C}_{3}\right) /$ Total, where Total is defined as the number of positions with genotype calls by both technologies, or $\left(\mathrm{A} 1+\mathrm{A} 2+\mathrm{A} 3+\mathrm{B} 1+\mathrm{B} 2+\mathrm{B}_{3}+\mathrm{C}_{1}+\mathrm{C}_{2}\right.$ $+\mathrm{C}_{3}$ ). Because the sequencing accuracy metric is dominated by the concordance of a large number of homozygous reference calls (A1), this metric tends to be very near 1 .

\section{Variant accuracy}

Because 'sequencing accuracy' tends to be dominated by the large number of homozygous reference calls, we define another metric called 'variant accuracy'. Variant accuracy is restricted to the variant positions called by $\mathrm{ABI}$ Sanger and is defined as: $\left(\mathrm{B}_{2}+\mathrm{C}_{3}\right) /\left(\mathrm{A}_{2}+\mathrm{A}_{3}+\mathrm{B}_{2}+\mathrm{B}_{3}+\mathrm{C}_{2}+\mathrm{C}_{3}\right)$.

Table I

Annotations of the genotypes differences to illustrate the definition of the metrics used to compare ABI Sanger and NGS Technologies Sanger

\begin{tabular}{lccc}
\cline { 2 - 4 } NGS technology & Homozygous reference & Heterozygous & Homozygous alternate \\
\hline Homozygous reference & Al & A2 & A3 \\
Heterozygous & BI & B2 & A4 \\
Homozygous alternate & Cl & C2 & B4 \\
N/N & DI & D2 & C4 \\
\hline
\end{tabular}

$\mathrm{N} / \mathrm{N}$ : positions at which genotype was not called. 
False positive rate of variants (false positive rate)

We define a false positive when the NGS technology calls a variant where ABI Sanger calls a homozygous reference. The false positive rate is calculated as $\left(\mathrm{B}_{1}+\mathrm{C}_{1}\right) /\left(\mathrm{B}_{1}+\mathrm{B}_{2}+\mathrm{B}_{3}+\right.$ $\left.\mathrm{C} 1+\mathrm{C}_{2}+\mathrm{C}_{3}\right)$.

False negative rate of variants (false negative rate)

We define a false negative when ABI Sanger detects a variant, but the NGS method calls this locus as a homozygous reference. The false negative rate is calculated as (A2 + $\left.\mathrm{A}_{3}\right) /\left(\mathrm{A}_{2}+\right.$ $\left.\mathrm{A}_{3}+\mathrm{B}_{2}+\mathrm{B}_{3}+\mathrm{C}_{2}+\mathrm{C}_{3}\right)$.

\section{Variant discrepancy rate}

We define the variant discrepancy rate as $\left(\mathrm{B}_{3}+\mathrm{C} 2\right) /\left(\mathrm{B} 2+\mathrm{B}_{3}\right.$ $\left.+\mathrm{C}_{2}+\mathrm{C}_{3}\right)$. This metric reflects ABI Sanger variant positions that are also detected by the NGS technology, but where the genotype calls disagree.

\section{Coverage rate}

The fraction of positions with genotype calls is defined as 1$\left(\mathrm{D} 1+\mathrm{D} 2+\mathrm{D}_{3}\right) /\left(\mathrm{A} 1+\mathrm{A} 2+\mathrm{A}_{3}+\mathrm{B}_{1}+\mathrm{B}_{2}+\mathrm{B}_{3}+\mathrm{C}_{1}+\mathrm{C}_{2}+\mathrm{C}_{3}\right.$ $+\mathrm{D} 1+\mathrm{D} 2+\mathrm{D} 3)$.

\section{$A B I$ Sanger false positive rate}

We define a ABI Sanger false positive when ABI Sanger calls a variant but all three NGS technologies call the locus as homozygous reference. We assume the NGS technologies to be correct, and this was confirmed by re-inspection of the ABI Sanger traces. The ABI Sanger false positive rate is calculated as follows. The numerator is the number of loci that are called as homozygous reference by all three NGS technologies, but as a variant in ABI Sanger. In the denominator, we consider all positions that were called as variant by Sanger and also had a genotype call by all three NGS technologies.

\section{$A B I$ Sanger false negative rate}

We define a ABI Sanger false negative as a locus where the initial call by ABI Sanger is homozygous reference but all three NGS technologies detect a variant at this locus. In the numerator of the ABI Sanger false negative rate, we count the number of variant loci that are identified by all three NGS technologies but called as homozygous reference by ABI Sanger. We note that zygosity may not agree among the three NGS technologies, but if all three technologies identify a variant at the position, the locus is included (Supplemental Table 12 in Additional data file 1). The denominator represents the number of loci called as variant by all three NGS technologies (although the zygosity may differ).

\section{Validation of genotypes discordant between ABI Sanger and the three NGS platforms}

PCR reactions were performed in $50 \mu$ platinum buffer (Invitrogen, Carlsbad, CA, USA) using 5 pM of primers (Supplemental Table 15 in Additional data file 1) and $0.2 \mu \mathrm{l}$ of platinum Taq DNA polymerase, incubated 2 minutes at $94^{\circ} \mathrm{C}$ followed by 35 cycles at $30 \mathrm{~s}$ at $94^{\circ} \mathrm{C}, 30 \mathrm{~s}$ at $60^{\circ} \mathrm{C}$ and $30 \mathrm{~s}$ at $72^{\circ} \mathrm{C}$, followed by 5 minutes at $72^{\circ} \mathrm{C}$ for final elongation. PCR products were purified using QIAquick PCR purification columns (Qiagen, Hilden, Germany)) and sequenced in both directions using the same primers as in the PCR and Big Dye terminator sequencing chemistry (Applied Biosystems, Foster City, CA USA).

\section{Simulations}

Simulations were performed in order to assess performance of each NGS platform at lower coverage depths. For each simulation, we randomly sampled a subset of the reads and recalled genotypes. The size of the subset was determined by the desired coverage depth.

\section{Inferring coverage at various error rate degradations}

To obtain the coverage depths in Figure 5, we first examined the error rate at the maximal simulated coverage. For 50\% error rate degradation, we multiplied the error rate at the maximal coverage by 1.5 to get the desired error rate. For $10 \%$ error rate degradation, we multiplied the error rate at the maximal coverage by 1.1 to get the desired error rate. We then examined the error rates from the simulations at different coverage depths, and interpolated what coverage depth corresponds to the desired error rate. For example, the false positive error rate for Illumina GA at $140 \times$ from the simulations is 0.073 . At $50 \%$ error rate degradation, the false positive rate is 0.110 . The false positive rates at coverage depths of $60 \times$ and $80 \times$ are 0.118 and 0.099 , respectively, so we know that a coverage depth within the range of $60 \times$ to $80 \times$ will give a false positive rate of 0.110 . Using linear interpolation, we deduce that a coverage depth of $68 \times$ gives a false positive rate of o.110, and this is reported in Figure 5 .

The error rates for Illumina GA and ABI SOLiD at maximum simulated coverage are slightly higher than what was experimentally observed. The additional errors observed in the simulations are largely associated with low coverage regions and are different between iterations, whereas the systematic errors present in the experimental data set at full coverage are shared between iterations. This shows that the simulation produces random sampling errors, directly associated with low coverage regions.

\section{Abbreviations}

AARF: alternate allele read frequency; LR-PCR: long-range PCR; NGS: next generation sequencing; SNP: single nucleotide polymorphism.

\section{Competing interests}

PCN and SS Murray currently hold stock in Illumina, Inc. 


\section{Authors' contributions}

$\mathrm{OH}, \mathrm{PN}$, and SL performed next gen sequencing analysis; SL, and TS performed Sanger sequencing analysis; XW, and KB performed next gen sequencing experiments; KF, ET, NS, RS, SL, and SM facilitated the study; KF, SL, PN, and $\mathrm{OH}$ designed the study and wrote the manuscript.

\section{Additional data files}

The following additional data are available with the online version of this paper: a PDF including 15 supplemental tables (Additional data file 1); supplemental Materials and methods (Additional data file 2); a PDF including three supplemental figures (Additional data file 3).

\section{Acknowledgements}

We thank Drs. Kevin McKernan and Stephen McLaughlin for providing assistance in the amplicon library preparation and initial data analysis for $A B I$ SOLiD sequence data, and Dr Dan Roden for permission to use $A B I$ Sanger trace data that were previously generated by the JCVI under the support of the National Heart, Lung and Blood Re-sequencing and Genotyping program [34]. This work was partly funded by NIH CTSA grant NIH IU54RR025204-0I.

\section{References}

I. Sanger F, Nicklen S, Coulson AR: DNA sequencing with chainterminating inhibitors. Proc Natl Acad Sci USA 1977, 74:5463-5467.

2. Bonetta L: Genome sequencing in the fast lane. Nat Methods 2006, 3:|4|-|47.

3. von Bubnoff $A$ : Next-generation sequencing: the race is on. Cell 2008, 132:72I-723.

4. Schuster SC: Next-generation sequencing transforms today's biology. Nat Methods 2008, 5:16-18.

5. Johnson DS, Mortazavi A, Myers RM, Wold B: Genome-wide mapping of in vivo protein-DNA interactions. Science 2007, 316:1497-1502.

6. Bhinge AA, Kim J, Euskirchen GM, Snyder M, lyer VR: Mapping the chromosomal targets of STATI by sequence tag analysis of genomic enrichment (STAGE). Genome Res 2007, 17:910-916.

7. Mortazavi A, Williams BA, McCue K, Schaeffer L, Wold B: Mapping and quantifying mammalian transcriptomes by RNA-Seq. Nat Methods 2008, 5:621-628.

8. Wang ET, Sandberg R, Luo S, Khrebtukova I, Zhang L, Mayr C, Kingsmore SF, Schroth GP, Burge CB: Alternative isoform regulation in human tissue transcriptomes. Nature 2008, 456:470-476.

9. Wheeler DA, Srinivasan M, Egholm M, Shen Y, Chen L, McGuire A, He W, Chen YJ, Makhijani V, Roth GT, Gomes X, Tartaro K, Niazi F, Turcotte CL, Irzyk GP, Lupski JR, Chinault C, Song XZ, Liu Y, Yuan Y, Nazareth L, Qin X, Muzny DM, Margulies M, Weinstock GM, Gibbs RA, Rothberg JM: The complete genome of an individual by massively parallel DNA sequencing. Nature 2008, 452:872-876.

10. Bentley DR, Balasubramanian S, Swerdlow HP, Smith GP, Milton J, Brown CG, Hall KP, Evers DJ, Barnes CL, Bignell HR, Boutell JM, Bryant J, Carter RJ, Keira Cheetham R, Cox AJ, Ellis DJ, Flatbush MR, Gormley NA, Humphray SJ, Irving LJ, Karbelashvili MS, Kirk SM, Li H, Liu X, Maisinger KS, Murray LJ, Obradovic B, Ost T, Parkinson ML, Pratt MR, et al: Accurate whole human genome sequencing using reversible terminator chemistry. Nature 2008, 456:53-59.

II. Wang J, Wang W, Li R, Li Y, Tian G, Goodman L, Fan W, Zhang J, Li J, Guo Y, Feng B, Li H, Lu Y, Fang X, Liang H, Du Z, Li D, Zhao Y, Hu Y, Yang Z, Zheng H, Hellmann I, Inouye M, Pool J, Yi X, Zhao J, Duan J, Zhou $Y$, Qin J, et al:: The diploid genome sequence of an Asian individual. Nature 2008, 456:60-65.

12. Hodges E, Xuan Z, Balija V, Kramer M, Molla MN, Smith SW, Middle CM, Rodesch MJ, Albert TJ, Hannon G], McCombie WR: Genome- wide in situ exon capture for selective resequencing. Nat Genet 2007, 39: I522-1527.

13. Albert TJ, Molla MN, Muzny DM, Nazareth L, Wheeler D, Song X, Richmond TA, Middle CM, Rodesch MJ, Packard CJ, Weinstock GM, Gibbs RA: Direct selection of human genomic loci by microarray hybridization. Nat Methods 2007, 4:903-905.

14. Okou DT, Steinberg KM, Middle C, Cutler DJ, Albert T], Zwick ME: Microarray-based genomic selection for high-throughput resequencing. Nat Methods 2007, 4:907-909.

15. Porreca GJ, Zhang K, Li JB, Xie B, Austin D, Vassallo SL, LeProust EM, Peck BJ, Emig CJ, Dahl F, Gao Y, Church GM, Shendure J: Multiplex amplification of large sets of human exons. Nat Methods 2007, 4:93I-936.

16. Jurka J: Repbase Update: a database and an electronic journal of repetitive elements. Trends Genet 2000, 16:418-420.

17. Dohm JC, Lottaz C, Borodina T, Himmelbauer H: Substantial biases in ultra-short read data sets from high-throughput DNA sequencing. Nucleic Acids Res 2008, 36: el 05.

18. Hillier LW, Marth GT, Quinlan AR, Dooling D, Fewell G, Barnett D, Fox P, Glasscock Jl, Hickenbotham M, Huang W, Magrini VJ, Richt RJ, Sander SN, Stewart DA, Stromberg M, Tsung EF, Wylie T, Schedl T, Wilson RK, Mardis ER: Whole-genome sequencing and variant discovery in C. elegans. Nat Methods 2008, 5:183-188.

19. Quinlan AR, Marth GT: Primer-site SNPs mask mutations. Nat Methods 2007, 4:192.

20. Stephens M, Sloan JS, Robertson PD, Scheet P, Nickerson DA: Automating sequence-based detection and genotyping of SNPs from diploid samples. Nat Genet 2006, 38:375-38I.

2I. Bhangale TR, Rieder MJ, Livingston RJ, Nickerson DA: Comprehensive identification and characterization of diallelic insertiondeletion polymorphisms in $\mathbf{3 3 0}$ human candidate genes. Hum Mol Genet 2005, 14:59-69.

22. Cronn R, Liston A, Parks M, Gernandt DS, Shen R, Mockler T: Multiplex sequencing of plant chloroplast genomes using Solexa sequencing-by-synthesis technology. Nucleic Acids Res 2008, 36:el22.

23. Margulies M, Egholm M, Altman WE, Attiya S, Bader JS, Bemben LA Berka J, Braverman MS, Chen YJ, Chen Z, Dewell SB, Du L, Fierro JM, Gomes XV, Godwin BC, He W, Helgesen S, Ho CH, Irzyk GP, Jando SC, Alenquer ML, Jarvie TP, Jirage KB, Kim JB, Knight JR, Lanza JR, Leamon JH, Lefkowitz SM, Lei M, Li J, et al.: Genome sequencing in microfabricated high-density picolitre reactors. Nature 2005 , 437:376-380

24. ABI-SOLID_specifications [http://www3.appliedbiosys tems.com/cms/groups/mcb_marketing/documents/generaldocu ments/cms_057562.pdf]

25. Illumina-GA_specifications [http://www.illumina.com/down loads/GenomeAnalyzer_SpecSheet.pdf]

26. Bhangale TR, Rieder MJ, Nickerson DA: Estimating coverage and power for genetic association studies using near-complete variation data. Nat Genet 2008, 40:84I-843.

27. Bhangale TR, Stephens M, Nickerson DA: Automating resequencing-based detection of insertion-deletion polymorphisms. Nat Genet 2006, 38: |457- | 462.

28. Ball EV, Stenson PD, Abeysinghe SS, Krawczak M, Cooper DN, Chuzhanova NA: Microdeletions and microinsertions causing human genetic disease: common mechanisms of mutagenesis and the role of local DNA sequence complexity. Hum Mutat 2005, 26:205-2I3.

29. Levy S, Sutton G, Ng PC, Feuk L, Halpern AL, Walenz BP, Axelrod N, Huang J, Kirkness EF, Denisov G, Lin Y, MacDonald JR, Pang AW, Shago M, Stockwell TB, Tsiamouri A, Bafna V, Bansal V, Kravitz SA, Busam DA, Beeson KY, Mclntosh TC, Remington KA, Abril JF, Gill J, Borman J, Rogers YH, Frazier ME, Scherer SW, Strausberg RL, et al:: The diploid genome sequence of an individual human. PLOS Biol 2007, 5:e254.

30. Craig DW, Pearson JV, Szelinger S, Sekar A, Redman M, Corneveaux JJ, Pawlowski TL, Laub T, Nunn G, Stephan DA, Homer N, Huentelman MJ: Identification of genetic variants using bar-coded multiplexed sequencing. Nat Methods 2008, 5:887-893.

3I. Smith DR, Quinlan AR, Peckham HE, Makowsky K, Tao W, Woolf B, Shen L, Donahue WF, Tusneem N, Stromberg MP, Stewart DA, Zhang L, Ranade SS, Warner JB, Lee CC, Coleman BE, Zhang Z, McLaughlin SF, Malek JA, Sorenson JM, Blanchard AP, Chapman J, Hillman D, Chen F, Rokhsar DS, McKernan KJ, Jeffries TW, Marth GT, Richardson PM: Rapid whole-genome mutational profiling using next-generation sequencing technologies. Genome Res 2008, I 8:1638-1642. 
32. Coriell Institute [http://www.coriell.org]

33. Li H, Ruan J, Durbin R: Mapping short DNA sequencing reads and calling variants using mapping quality scores. Genome Res 2008, I 8: | 85|-|858.

34. National Heart, Lung and Blood Re-sequencing and Genotyping program [http://rsng.nhlbi.nih.gov/] 